\title{
QUERCETIN AND CURCUMIN PREVENT DECREASING OF LDL-CHOLESTEROL AND INCREASING OF HDL- CHOLESTEROL IN HIGH FAT DIET RATS
}

\author{
Made Harumi $\mathrm{P}^{1}$, Gita Trisna ${ }^{1}$, Nyoman Odiyana $\mathrm{P} \mathrm{G}^{1}$, \\ Deby Aulia ${ }^{1}$, AAA Putri Khrisnawati ${ }^{1}$, Arta Farmawati ${ }^{2}$, \\ Medicine Study Program of Faculty of Medicine Universitas Gadjah Mada ${ }^{1}$ \\ Department of Biochemistry Faculty of Medicine Universitas Gadjah Mada ${ }^{2}$ \\ Email:harumipadmaswari@gmail.com
}

\begin{abstract}
Cardiovascular disease is number one killer disease in the world. The most common etiology is atherosclerosis. LDL and HDL-cholesterol are often associated with atherosclerosis which are affected by diet. Quercetin and curcumin considered have influenced to $L D L$ and HDL. They are antioxidant that protect against LDL oxidation and raised HDL-cholesterol. In this study, we invest about comparison effect of prevention in LDL and HDL level change, after giving quercetin and curcumin. 20 males Rattus novergicus rats in 200-300 grams divided into 4 groups. Group B, $C, D$ were given high-fat diet for 4 weeks, while group $A$ (negative control) was given standard woof. Group $B$ was positive control and no treatment, $C$ was given quercetin, $D$ was given curcumin. The treatment and giving high-fat diet was done simultaneously to detect prevention effect. HDL and LDL-cholesterol evaluated two times, pre and post-treatment. Result showed the increase prevention level of LDL-cholesterol and decrease prevention level of $H D L$-cholesterol in group $C$ and $D$ significantly. The best prevention effect is quercetin, $p=0.000$. Furthermore, we showed there was no significant correlation between $\triangle L D L$ and $\triangle H D L$-cholesterol level. In conclusion, quercetin and curcumin have a good effect in preventing hypercholesterolemia, however quercetin have better effect than curcumin.
\end{abstract}

Keywords: quercetin curcumin, HDL-cholesterol, LDL-cholesterol

\section{INTRODUCTION}

Cardiovascular

disease

(CVD) is the most common cause of

deaths worldwide, about $80 \%$ of the burden occurs in developing countries (Kumar et al., 2010). CVD includes all diseases in the heart and blood vessels. WHO

(2008) estimated 17.3 million people died by CVD, about 7.3 million were due to coronary heart disease and 6.2 million were due to stroke. Atherosclerosis is one of common causes of stroke. 
Atherosclerosis is the most common pathological feature of vascular obstruction resulting in thrombotic stroke. It is characterized by intimal lesions called atheromas that protrude into vessel lumens. Atherosclerosis formation depends on lipid level in the blood. Hypercholesterolemia is a major risk factor for atherosclerosis even in the absence of other factors. The major component of serum cholesterol associated with increased risk of atherosclerosis event is low density lipoprotein cholesterol (LDL-C). LDL-C is the form of cholesterol that is delivered to peripheral tissues. In contrast, high density lipoprotein cholesterol (HDL-C) mobilizes cholesterol from tissue and transports it to the liver to be excreted in the bile. Consequently, higher levels of HDL-C correlate with reduced risk (Kumar et al., 2010).

Atherosclerosis can be prevented by controlling the risk factors, particularly such as cigarette smoking, unhealthy diet, physical inactivity, high blood pressure, diabetes, and raised lipids. Lipid levels in the blood are affected by diet including additional diet, such as antioxidant that can reduce LDL-C levels and increase HDL-C levels. It prevents targeted molecule to be oxidized by free radical. According to the oxidative modification hypothesis, Wang et al. (2012) said oxidation of LDL is crucial to the cellular uptake of LDL in the first stage of atherosclerotic plaque development. Because of that reason, antioxidant plays vital role in preventing atherosclerosis.

Curcumin and quercetin are antioxidants that have not been widely used. Curcumin has pharmacological effects including antitumor and anti-inflammation effects and can prevent lipid peroxidation. Sudjarwo (2011) concludes that curcumin can repair endothelium-dependent dilation at blood vessels wall and reduce lipid peroxidation in aorta wall. Quersetin is a flavonoid that can also affect lipid levels in the blood by reducing LDL-C concentrations. Flavonoids, particularly quercetin has been associated with a decreased risk of CVD. Quersetin can be found in onion, green tea, and apple. Based on 
the effect of each compound, combination of both quercetin and curcumin are expect to be more effective in managing hypercholesterolemia and in preventing atherosclerosis. This study compares quarcetin and curcumin, in preventing hypercholesterolemia.

\section{METHOD}

Chemicals

Quercetin and $\mathrm{Na}-\mathrm{CMC}$ were of analytical grade obtained from Sigma Aldrich Chemical and the curcumin were procured from Doctor Eko Wahjuni (POM TR 023217 101).

Animals

Animal experiments were carried out taking appropriate measures to minimize pain or discomfort with due clearance from the Medical and Health Research Ethics Committee, Faculty of Medicine Universitas Gadjah Mada (UGM)

Male wistar rats (Rattus novergicus) weighing between 200$300 \mathrm{~g}$ each were used for this experiment. They were procured from PAU Laboratory UGM, Yogyakarta. The rats were maintained in a controlled environment under standard condition of temperature $\left(28 \pm 2^{\circ} \mathrm{C}\right)$ and humidity with an alternating light and dark cycle. The animals were housed in individual stainless steel cages were maintained on various experimental diets ad libitum. The basal diet used was AD2 woof and the hypercholesterolemic diet consisted of AD-2 woof plus cow's fat which administered by sonde.

Experimental Procedure

After a week of acclimatization, the rats were divided into four groups with 5 rats in each group. Group I served as negative control (basal diet+1 $\mathrm{mL} \mathrm{Na}-\mathrm{CMC}$ $0.5 \%$ ), Group 2 served as positive control (hypercholesterolemic diet+1 $\mathrm{mL} \mathrm{Na}-\mathrm{CMC}$ 0.5\%), Group 3 treated with hypercholesterolemic diet+ $45 \mathrm{mg} / \mathrm{kgBW}$ quercetin+1 $\mathrm{mL} \mathrm{Na}-$ CMC $0.5 \%$, Group 4 treated with hypercholesterolemic diet +45 $\mathrm{mg} / \mathrm{kgBW}$ curcumin $+1 \mathrm{~mL} \mathrm{Na}-\mathrm{CMC}$ $0.5 \%$. After 4 weeks of treatment period, the animals were sacrificed 
under light ether anesthesia. Blood was collected by orbital artery for two times. First, at the end of acclimatization, and second at the end of experiment. Blood was separated from serum by sentrifugation and used for lipid analysis.

Lipid Profile

HDL and LDL-cholesterol were determined by homogenous enzymatic colorimetric assay method.

Statistical Analysis

Result are expressed as means $\underline{+} \mathrm{SD}$ and comparison between groups were made by means of oneway ANOVA. Comparison among different groups were made applying the Tukey Post Hoc Test and were considered significant when $\mathrm{p}<0.05$.

\section{RESULT AND DISCUSSION}

As observed in this study, high cholesterol feeding for 4 weeks resulted in a significant increase in LDL-cholesterol concentration and a decrease in HDL-cholesterol (Table 1 and 2). The increased in LDLcholesterol and decreased in HDLcholesterol were variously. Dietary quercetin or curcumin prevented the increase of LDL-cholesterol significantly. However, quercetin prevented better than curcumin.

Table I. Effect of quercetin and curcumin in HDL-cholesterol

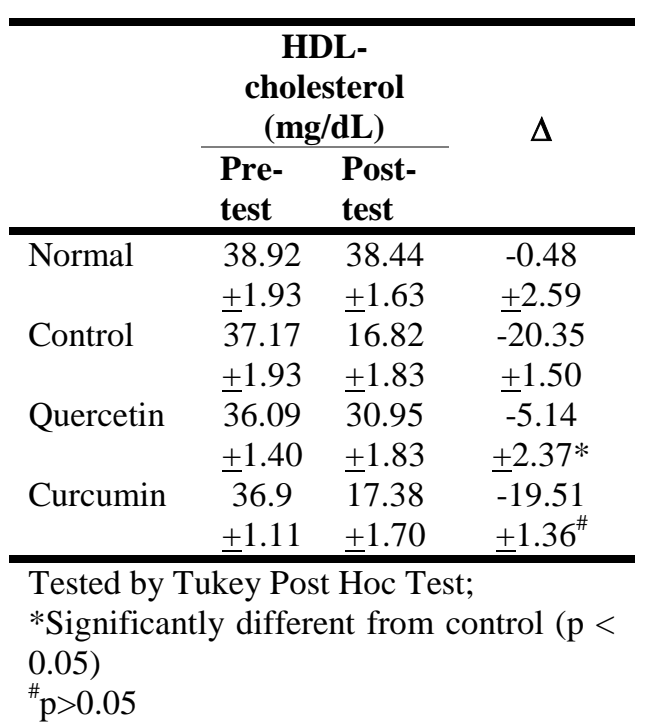

HDL-cholesterol essentially decrease in all group significantly. Quercetin was prevented the decrease of HDL-cholesterol significantly in compared to control.

As shown in Table 3, there was no significant correlation between increased in LDL-cholesterol with decreased in HDL-cholesterol. Data shows that quercetin or curcumin treated group had a very weak positive correlation. It means the increased in LDL-cholesterol make HDL-cholesterol slightly increased. 
Table II. Effect of quercetin and curcumin in LDL-cholesterol

\begin{tabular}{llll}
\hline & \multicolumn{2}{l}{ LDL-cholesterol $(\mathbf{m g} / \mathbf{d L})$} & \multirow{2}{*}{$\Delta$} \\
\cline { 2 - 3 } & Pre-test & Post-test & \\
\hline Normal & $50.16 \pm 2.38$ & $53.11 \pm 2.35$ & $2.94 \pm 0.36$ \\
Control & $45.02 \pm 2.31$ & $89.76 \pm 2.52$ & $44.75 \pm 3.42$ \\
Quercetin & $48.84 \pm 6.28$ & $63.54 \pm 2.99$ & $14.7 \pm 8.99 *$ \\
Curcumin & $44.35 \pm 7.47$ & $78.39 \pm 1.73$ & $34.04 \pm 8.36^{*}$ \\
\hline
\end{tabular}

Tested by Tukey Post Hoc Test;

*Significantly different from control $(\mathrm{p}<0.05)$

Table III. Correlation between increased in LDL and decreased in HDL-cholesterol

\begin{tabular}{lll}
\hline & $\mathbf{r}$ & $\mathbf{p}$ \\
\hline Normal & -0.864 & 0.059 \\
Control & -0.146 & 0.815 \\
Quercetin & 0.219 & 0.723 \\
Curcumin & 0.123 & 0.843 \\
\hline
\end{tabular}

Tested by Pearson Correlation;

Significantly different if $\mathrm{p}<0.05$

Epidemiological studies have reported a reduced risk of coronary heart disease in subjects with high flavonoid intake (Knekt et al., 1995). The protective effect of flavonoids has been attributed to many mechanisms, i.e. antioxidant properties, anti-inflammatory activity (Miller A, 1996), antiproliferative as well as antipatelet effects (Moghaddam et al., 2012; Guerrero et al., 2005).

In the present study, hypolipidemic effects was evaluated in two substance. It was demonstrated that all treatment groups showed a positive effect in the prevention of hyper-cholesterolemia, which prevents the increase in LDL as well as prevent a significant decrease in HDL (Table I and II).

These results are consistent with the theory presented by Srinivasan et al. (2004) which states that curcumin has hypolipidemic activity, by increasing receptor LXR (liver X receptor) and RXR (retinoic $\mathrm{X}$ receptor), which causes an increase in metabolism of cholesterol, especially LDL uptake increase. Although it has an excellent effect on hypolipidemic, the major 
concern of curcumin is a limited bioavailability which limits its therapeutic utility (Anand et al. 2007).

Earlier studies have shown that quercetin significantly inhibit in vitro LDL oxidation, and also protects macrophages from oxidized lowdensity lipoprotein induced apoptosis (Naderi et al., 2003; Yao et al., 2012). Quercetin acts to inhibit atherosclerosis through up-regulation of reverse cholesterol transport, so as to reduce cholesterol levels in the blood (Fernandes et al., 2012). Quercetin inhibits sulfotransferase enzymes and also limited by poor bioavailability though it's better than curcumin (Kaur G \& Meena C, 2012).

The mechanisms of each compounds lead to the prevention effects of hypercholesterolemia. However, based on this study, quercetin was more impressive effect. Quercetin was effective in prevent hypercholesterolemia by high-fat diet, after 4-weeks of the experiment. While this activity was less prominent in the curcumin treatment.

It is well known that HDLcholesterol levels have a protective role in cardiovascular disease (Silbernagel et al., 2013). Similarly increased level of LDL-cholesterol results in increased risk of development of atherosclerosis (Warnholtz et al., 2001). However, the study exhibited that there was no significant correlation between increases in LDL-cholesterol with decreases in HDL-cholesterol.

The anti-atherogenic properties were described to the antioxidant activity measured by the reduced formation of LDL-cholesterol and enhaced formation of HDLcholesterol. Quercetin in dose $45 \mathrm{mg} / \mathrm{kgBW}$ prevented hypercholesterolemia optimal, although it had not reached normal conditions.

\section{CONCLUSION}

The quercetin in $45 \mathrm{mg} / \mathrm{kgBW}$ dose is the best treatment in this study, because the maximum preventive effect. Preventive effects of hypercholesterolemia slowing the formation of fatty streak which is 
going from atherosclerosis, the biggest risk factor for cardiovascular diseases.

\section{ACKNOWEDGEMENT}

This study was supported by

Directory of Higher Education in Indonesia (DIKTI) grant 2014.

\section{REFERENCE}

Anand P, et al., 2007, Bioavailability of Curcumin: Problems and Promises, Mol. Pharmaceutics, 4 (6), 807-818.

Fernandes MM, et al., 2012, Physical exercise and microRNAs: new frontiers in heart failure, Arq Bras Car, 98(5):459-466.

Guerrero JA, et al., 2005, Flavonoids inhibit platelet function through binding to the thromboxane A2 receptor, $J$ Thromb Haemost, 3: 369-376.

Kaur G and Meena C., 2012, Amelioration of Obesity, Glucose Intolerance, and Oxidative Stress in High-Fat Diet and Low-Dose Streptozotocin-Induced Diabetic Rats by Combination Consisting of Curcumin with Piperine and Quercetin, ISRN Pharmacology, 2012:1-7.

Knekt P, et al., 1995, Flavonoid intake and long-term risk of coronary heart disease and cancer in the Seven Countries Study, Arch Intern Med, 155: 381-386.
Kumar V, et al., 2010, Robbin and Cotran Pathology Basic of Disease, $8^{\text {th }}$ ed, Philadhelpia: Saunders Elsevier.

Miller A., 1996, Antioxidant Flavonoids: Structure, Function and Clinical Usage, Alt Med Rev, 1(2):103-111.

Moghaddam G, et al., 2012, Antiproliferative Activity of Flavonoids: Influence of the Sequential Methoxylation State of the Flavonoid Structure, Phyto Res, 26(17):1023-1028.

Naderi GA, et al., 2003, Anti-oxidant effect of flavonoids on the susceptibility of LDL oxidation, Mol Cell Biochem, 246(1-2):193-196.

Silbernagel G, et al., 2013, Highdensity lipoprotein cholesterol, coronary artery disease, and cardiovascular mortality, Eur Heart $J$ http://eurheartj.oxfordjournals org/content/early/2013/09/07/e urheartj.eht343 was accessed on December 2, 2014.

Srinivasan K, Sambaiah K, Chandrasekhara N., 2004, Spices as beneficial hypolipidemic food adjuncts: a review, Food Rev Int, 20: 187220.

Sudjarwo, SA., 2011, Mechanisms of endothelial cell protection by quercetin in hypercholesterolemia, $J$ App Phar Sci, 3:123-127.

Wang L, et al., 2012, Protective Effect of Polyphenol Extract of Adlay (Coix lachryma-jobi L. var. ma-yuen Stapf) on Hypercholesterolemia- induced Oxidative Stress in Rats, Molecules, 17: 8886-8897. 
Warnholtz, A et al., 2001, Antioxidants and endothelial dysfunction in hyperlipidemia, Curr Hypertens Rep, 3:53-60.

World Health Organization., 2008, Cardiovascular Disease: Global Atlas on Cardiovascular Disease Prevention and Control, Geneva, Switzerland: WHO,

http://www.who.int/mediacentr e/factsheets/fs317/en/ was accessed on December 6, 2014

Yao S, et al., 2012, Quercetin protects macrophages from oxidized low-density lipoproteininduced apoptosis by inhibiting the endoplasmic reticulum stress-C/EBP homologous protein pathway, Exp Biol Med, 237(7):822-831 